\title{
Molecular identification of Monascus purpureus NART001 isolated from commercially available Chinese red fermented rice
}

\author{
Identificação molecular de Monascus purpureus NART001 isolado do arroz vermelho \\ fermentado Chinês disponível comercialmente
}

\author{
Nelson Tavares ${ }^{1}$, Pedro Miguel Penedo ${ }^{2}$ \\ ${ }^{1}$ CBIOS - Research Center for Biosciences and Health Technologies, Universidade Lusófona de Humanidade e Tecnologias, \\ Campo Grande 376, 1649-024 Lisboa, PORTUGAL \\ ${ }^{2}$ DNA Sequencing Laboratory, Stabvida, Madan Parque, 2825-182 Caparica, PORTUGAL \\ Email: nelson.tavares@ulusofona.pt
}

\begin{abstract}
The Horizon 2020 document emphasizes that the most important goals and objectives for common research programmes are to address major global challenges. Priorities are the improvement of human health, combating life-style diseases, and ensuring food security for a rapidly growing population. Yeast rice is a product obtained from rice fer-mented by a red yeast, Monascus purpureus. The medicinal properties of red yeast rice have a favourable impact on lipid profiles of hypercholesterolemic persons. The aim of this study was to molecularly identify an isolate of a strain of Monascus purpureus (NART001) obtained previously. Following an established protocol, DNA of the sample was isolated and amplified with specific primers and was confirmed using an electrophoresis system. The Basic Local Alignment Search Tool was used for species identification. Results of investigation revealed that the Monascus purpureus NART001 strain showed 100\% identity with Monascus purpureus.
\end{abstract}

Keywords: Monascus purpureus, Red yeast rice, molecular, identification

\section{Resumo}

O Documento Horizonte 2020 sublinha que as metas e os objectivos mais importantes para os Programas de Investigação da Comunidade Europeia são abordar os principais desafios globais. Entre os desafios prioritários estão a melhoria da saúde humana, combater doenças do estilo de vida e garantir a segurança alimentar para uma população em rápido crescimento. $\mathrm{O}$ arroz vermelho fermentado é um produto obtido a partir de arroz fermentado pelo Monascus purpureus. As propriedades medicinais do arroz vermelho fermentado têm um impacto favorável nos perfis lipídicos de pessoas hipercolesterolémicas. O objetivo deste estudo é identificar uma estirpe isolada de Monascus purpureus NART001 obtida anteriormente, seguindo um protocolo estabelecido no isolamento e amplificação do ADN. Uma amostra de ADN foi isolada e amplificada com primers específicos e foi confirmada utilizando o sistema de electroforese. A Basic Local Alignment Search Tool foi utilizada para a identificação da estirpe. Os resultados revelaram que a estirpe Monascus purpureus NART001 apresentou 100\% de identidade com Monascus purpureus.

Palavras-Chave: Monascus purpureus, Arroz vermelho fermentado, identificação molecular 


\section{Introduction}

The vision of the European common research programme for 2014-2020, called Horizon 2020, is to create a smarter, more sustainable and more inclusive society. However, this is a global endeavor, and is important for mycologists all over the world because it includes a special role for fungi and fungal products. The Horizon 2020 document emphasizes that the most important goals and objectives for common research programmes are to address the major global challenges. Among the challenges of priority is the improvement of human health, combating life-style diseases, and ensuring food security for a rapidly growing population. Fungi and fungal products are also instrumental in producing fermented foods to improve health (1). Monascus purpureus fermentation products have been widely used by people primarily in Asian countries for many centuries as food colourant, preservative and in traditional medicine (2). The medicinal properties of red yeast rice have a favourable impact on lipid profiles of hypercholesterolemic patients $(3,4)$. Red yeast rice consists of multiple bioactive substances, including monacolin $\mathrm{k}$, which is chemically identical to lovastatin, has been recognized as responsible for the cholesterol reducing effect of this compound (5). For the first time Monascus purpureus NART001 strain was successfully isolated by surface sterile technique as described in a previous study (6). Inoculation and cultivation of isolated Monascus purpureus NART001 were performed using glutinous rice Oryza sativa L. The obtained strain showed macroscopic characteristics, such as colour and colony shape, and microscopic features, with similarity to Monascus purpureus. As an alternative to traditional morphology-based species delimitation, the identification of a new fungal species could be made using differences in DNA sequence data. With modern approaches based on DNA sequences, it is possible to accurately identify unknown fungal species (7). DNA analysis offers the potential benefits of high sensitivity and rapid detection (8). The species identification of fungi has been based primarily on the use of variable ribosomal-DNA (rDNA) internally transcribed spacer (ITS) regions, which generally provide greater taxonomic resolution than those from coding regions (9). The aim of the present study was to perform the DNA identification of the strain using DNA sequencing analysis for molecular species identification.

\section{Introdução}

A visão do Programa de Investigação da Comunidade Europeia para 2014-2020, denominado Horizonte 2020, é criar uma sociedade mais inteligente, mais sustentável e mais inclusiva. No entanto, este é um esforço global, que também é importante para micologistas em todo o mundo, porque inclui um papel especial para fungos e produtos fúngicos. O Documento Horizonte 2020 sublinha que as metas e os objectivos mais importantes para os Programas de Investigação Comunitária são abordar os principais desafios globais. Entre os desafios de prioridade estão a melhoria da saúde humana, combater doenças de estilo de vida e garantir a segurança alimentar para uma população em rápido crescimento. Fungos e produtos fúngicos também são instrumentais na produção de alimentos fermentados para melhorar a saúde (1). Os produtos de fermentação de Monascus purpureus têm sido amplamente utilizados, por pessoas principalmente nos países asiáticos, durante muitos séculos como corantes alimentares, conservantes e na medicina tradicional (2). As propriedades medicinais do arroz vermelho fermentado têm um impacto favorável nos perfis lipídicos de pacientes hipercolesterolémicos $(3,4)$. $\mathrm{O}$ arroz vermelho fermentado é constituído por várias substâncias bioactivas, incluindo a monacolina $\mathrm{k}$, que é quimicamente idêntica à lovastatina, que tem sido reconhecida como a responsável pelos efeitos de redução do colesterol (5). Pela primeira vez a estirpe NART001 de Monascus purpureus foi isolada com sucesso por técnica estéril de superfície como descrito num estudo prévio (6). A inoculação e o cultivo do isolado de Monascus purpureus NART001 foram realizados utilizando arroz glutinoso Oryza sativa L. A estirpe obtida apresentou características macroscópicas, como cor e forma das colónia, assim como características microscópicas, semelhantes ao Monascus purpureus. Como alternativa à delimitação tradicional de espécies baseadas na morfologia, a identificação de uma nova espécie fúngica poderá ser feita observando diferenças nos dados de sequência de DNA. Com as abordagens modernas baseadas em sequências de DNA, é possível identificar com precisão espécies fúngicas desconhecidas (7). A análise de DNA oferece os potenciais benefícios da detecção altamente sensível e rápida (8). A identificação de espécies de fungos baseia-se principalmente na utilização de regiões variáveis de espaçador interno transcritas (ITS) de DNA ribossomal (rDNA) que geralmente proporcionam maior resolução taxonómica do que as regiões codificadoras (9). O objetivo do presente estudo foi realizar a identificação do DNA da estirpe, utilizando uma análise de sequenciamento de DNA para identificação de espécies moleculares. 


\section{Materials and Methods}

An axenic culture of Monascus purpureus NART001 was used. A colony of culture medium was minced with a sterile blade and suspended in $65 \mu 1$ of phosphate buffered saline. Three drops of the suspension were applied on a FTA ${ }^{\mathrm{TM}}$ Micro Indicating Card (GE Healthcare, USA), which was then left to dry at room temperature for two hours. The card was sent to STAB Vida (http://www.stabvida.com/pt/) for extraction, amplification, and sequencing of DNA.

A punch of $2 \mathrm{~mm}$ from the card containing the sample identified as ID029 was taken for the purification of nucleic acids using the FTA Reagent Purification reagent (GE Healthcare, USA). After the DNA was purified, PCR was performed directly on the card punch in a thermal cycler (8800 MyCycler, Agilent Technologies, USA). The reaction mixture was made using the Surf Hot Taq kit (STAB LIFE, Portugal), containing $10 \mathrm{x}$ PCR Buffer, $1.5 \mathrm{mM} \mathrm{MgCl}_{2}, 0,2 \mathrm{~mm}$ dNTP mix, 0.4 $\mu \mathrm{M}$ universal forward primer (27F- AGAGTTTGATCMTGGCTCAG) universal primer and reverse (1492RTACGGYTACCTTGTTACGACTT) and Surf Hot 2U Taq DNA polymerase. Milli-Q sterile water was used to bring the total volume to $25 \mu \mathrm{L}$ prior to initiation of the reaction. The sample was subjected to an initial denaturation of $95{ }^{\circ} \mathrm{C}$ for 15 minutes, followed by 35 cycles with a denaturation of $95^{\circ} \mathrm{C}$ for 30 seconds, annealing the $51^{\circ} \mathrm{C}$ for 30 seconds, and extension to $70^{\circ}$ $\mathrm{C}$ for 2 minutes. The final extension was $70{ }^{\circ} \mathrm{C}$ for 5 minutes. PCR products were purified using carboxylated magnetic beads (MCLab, USA) and the products were analyzed via agarose gel $(1.5 \%)$ in TAE 1 $\mathrm{x}$ buffer, and using the marker kit GeneRuler Express DNA Ladder. The gel was examined in a translluminator and via BOX5 VX5 (Vilbert Lourmat, France), with the aid of the intercalating agent GelRed (Biotium, USA), Figure 1. The primers used for sequencing the universal primers are indicated in Table 1. Sequencing was performed using the BigDye Terminator 3 kit (Applied Biosystems, USA) and the fragments were analyzed in 3730XL DNA Analyzer sequencer (Applied Biosystems, USA). Generated sequences were aligned using the Sequencher software 5.0 (GeneCodes, USA) in order to generate the consensus sequence. Consensus sequences were aligned against the nucleotide $\mathrm{Ba}-$ sic Local Alignment Search Tool (BLAST) database (10). With thistool, non-matching nucleotides from the standard nucleotide BLAST result against NCBI database would be indicated, and the highest identity value used to attribute the suggested species.

\section{Materiais e Métodos}

Utilizou-se uma cultura axénica de Monascus purpureus NART001. Uma colónia do meio de cultura foi picada com uma lâmina estéril e suspensa em $65 \mu \mathrm{L}$ de tampão fosfato-salino. Foram aplicadas 3 gotas da suspensão obtida num Micro Cartão FTA, e deixadas secar à temperatura ambiente durante 2 horas. A extração e amplificação de DNA, bem como o sequenciamento foi feito na STAB Vida (http://www.stabvida.com/pt/), para onde o cartão foi enviado.

Do Cartão FTA, foi retirado para a purificação de ácidos nucleicos uma punção de $2 \mathrm{~mm}$ identificada como amostra ID029 utilizando o reagente FTA Reagent Purification (GE Healthcare, EUA). Após a purificação do $\mathrm{ADN}$, a PCR foi realizada directamente na punção no termociclador 8800 MyCycler (Agilent Technologies, EUA). A mistura reaccional foi preparada utilizando o kit Surf Hot Taq (STAB LIFE, Portugal), contendo $10 \mathrm{x}$ tampão de PCR, 1,5 mM de $\mathrm{MgCl}$ 2, 0, 2 mm de mistura de dNTP, 0,4 $\mu \mathrm{M}$ de primer universal (27F- AGAGTTTGATCMTGGCTCAG) 1492R-TACGGYTACCTTGTTACGACTT) e Surf Hot 2U Taq DNA polimerase. Utilizou-se água estéril Milli-Q para completar um volume total de $25 \mu \mathrm{L}$ antes do início da reacção. A amostra foi submetida a uma desnaturação inicial a $95{ }^{\circ} \mathrm{C}$ durante 15 minutos, seguida de 35 ciclos com desnaturação a $95{ }^{\circ} \mathrm{C}$ por 30 segundos, anelamente a 51 ${ }^{\circ} \mathrm{C}$ por 30 segundos e extensão a $70{ }^{\circ} \mathrm{C}$ por 2 minutos. A extensão final foi de $70{ }^{\circ} \mathrm{C}$ durante 5 minutos. Os produtos da PCR foram purificados utilizando esferas magnéticas carboxiladas (MCLab, EUA) e analisados em gel de agarose $(1,5 \%)$ em tampão TAE 1 x e utilizando o marcador kit GeneRuler Express DNA Ladder. A foto do gel foi revelada em transluminador e-BOX5 VX5 (Vilbert Lourmat, França), com a ajuda do agente intercalante GelRed (Biotium, EUA) Figura 1. Os primers utilizados para sequenciar os primers universais são mencionados na Tabela 1. A sequenciação foi efectuada utilizando o kit BigDye Terminator 3 (Applied Biosystems, EUA) e os fragmentos foram analisados no 3730XL DNA Analyzer sequencer (Applied Biosystems, EUA). As sequências geradas foram alinhadas utilizando o software Sequencher 5.0 (GeneCodes, EUA), para gerar a sequência de consenso. As sequências de consenso foram alinhadas com a base de dados de nucleotídeo Basic Local Alignment Search Tool (BLAST) (10). O aparecimento de bases a negrito na tabela significa que existem nucleotídeos das sequências de consenso geradas que não estavam alinhados com a base de dados de nucleotídeos BLAST. Foi considerado o valor mais elevado para atribuição da espécie sugerida. 


\section{i. Agarose gel electrophoresis of the PCR products}
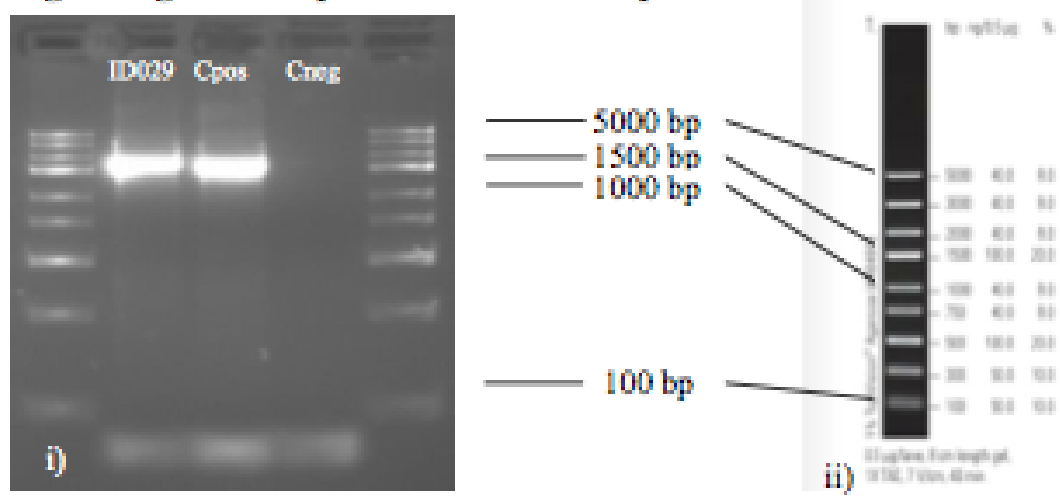

ii)

Figure 1/ Figura 1 - Agarose gel electrophoresis (1.5\%, 100v, 20 minutes) i) Agarose (1\%) gel electrophoresis of the PCR product. $1 \mu 1$ of PCR product was loaded into the gel. ii) DNA Ladder. $5 \mu \mathrm{l}$ of DNA Ladder was used in the agarose gel electrophoresis/ Electroforese em gel de agarose $(1,5 \%, 100 \mathrm{v}, 20$ minutos) i) Produto do PCR em gel de agarose (1\%). foi colocado $1 \mu 1$ do produto do PCR no gel. ii) DNA Ladder. $5 \mu 1$ of DNA Ladder foi usado na eletroforese em gel de agarose.

Table 1/ Tabela 1 - The primers used for sequencing the universal primers/ Primers utilizados na sequenciação dos primers universais.

\begin{tabular}{|l|l|}
\hline 27F & 5' - AGAGTTTGATCMTGGCTCAG - 3' \\
\hline $518 \mathrm{~F}$ & 5' - CCAGCAGCCGCGGTAATACG - 3' \\
\hline $800 \mathrm{R}$ & 5' $^{\prime}$ - TACCAGGGTATCTAATCC $-3^{\prime}$ \\
\hline $1492 \mathrm{R}$ & $5^{\prime}-$ TACGGYTACCTTGTTACGACTT - 3' \\
& \\
\hline
\end{tabular}




\section{Results}

All obtained bases for the studied sample indicate no non-matching nucleotides when compared with the nucleotides expected for Monascus purpureus (Table 2). The amplified DNA in the sample showed 100\% identity with Monascus purpureus (Table 3).

\section{Resultados}

Todas as bases apresentam-se uniformes (Tabela 2). O que sugere a inexistência de nucleotídeos não alinhados. A amostra ID029 apresentou 100\% de identidade com o Monascus purpureus (Tabela 3).

Table 2/ Tabela 2 - Consensus sequences obtained from sample sequencing generated data/ Sequências de consenso obtidas a partir de dados gerados pelo sequenciamento da amostra.

\begin{tabular}{|c|c|}
\hline Sample ID & FASTA (Consensus sequence) \\
\hline ID029 & $\begin{array}{l}\text { CCAACCTCCCACCCGTGATTATTGTACCTCCTGTTGCTTCGGCGCGGCCC } \\
\text { CCTGGGGCCCGCCGGAGACATCTTCTCGAACGCTGTCTTTGAAAAGGATTG } \\
\text { CTGTCTGAGTAAACATACCAAATCGGTTAAAACTTTCAACAACGGATCT } \\
\text { CTTGGTTCCGGCATCGATGAAGAACGCAGCGAAATGCGATAAGTAATGTG } \\
\text { AATTGCAGAATTCAGTGAATCATCGAATCTTTGAACGCACATTGCGCCCC } \\
\text { CTGGTATTCCGGGGGGCATGCCTGTCCGAGCGTCATTACTGCCCCTCAAGC } \\
\text { GCGGCTTGTGTGTTGGGCCGCCGTCCCCTGCGCCTCCGGGCAACGGGGACG } \\
\text { GGCCCGAAAGGCAGTGGCGGCGCCGCGTCCGGTCCTCGAGCGTATGGGGC } \\
\text { TTTGTCACCCGCTCAGTAGGTCGGGCCGGGGCCTTTCCCTCTCCAACCTTT } \\
\text { TTTTCCTTAGGTTGACCTCGGATCAGGTAGGGATACCCGCTGAACTTAAGC } \\
\text { ATATCAATAAGCGGAGGAAAAGAAACCAACCGGGATTGCCTCAGTAACGG } \\
\text { CGAGTGAAGCGGCAAGAGCTCAAATTTGAAAGCTGGCCCCTCCGGGGTCC } \\
\text { GCGTTGTAATTTGCAGAGGATGCTTCGGGCTCAGCCCCCGTCTAAGTGCCC } \\
\text { TGGAACGGGCCGTCGGAGAGGGTGAGAATCCCGTCTGGGACGGGGTGCCT } \\
\text { GGGTCCATGTGAAGCTCCTTCGACGAGTCGAGTTGTTTGGGAATGCAGCTC } \\
\text { TAAATGGGTGGTAAATTTCATCTAAAGCTAAATACTGGCCGGAGACCGAT } \\
\text { AGCGCACAAGTAGAGTGATCGAAAGATGAAAAGCACTTTGAAAAGAGAG } \\
\text { TTAAACAGCACGTGAAATTTTGAAAGGGAAGCGCTTGCGATCAGACTCG } \\
\text { CCTGCGGGGTTCAGCCGGCATTCGTGCCGGTGTACTTCCCCGTGGGCGGGC } \\
\text { CAGCGTCGGTTCGGGTGGCCGGTCAAAGGCCCCGGGAATGTGTCGCCCTC } \\
\text { CGGGGCGTCTTATAGCCCGGGGTGCCATGCGGCCTACCTGGACCGAGGAA } \\
\text { CGCGCTTCGGCTCGGACGCTGGCGTAATGGTCGTAAGCGAC }\end{array}$ \\
\hline
\end{tabular}

Table 3/ Tabela 3 - Suggested species of each consensus sequence BLASTed against NCBI nucleotide database/ Espécie sugerida como resultado da sequência submetida que é coberta pelo alinhamento por comparação com os nucleotídeos da base de dados NCBI.

\begin{tabular}{|c|c|c|c|}
\hline $\begin{array}{c}\text { Sample } \\
\text { ID/Amostra } \\
\mathrm{N}^{\mathrm{o}}\end{array}$ & $\begin{array}{c}\text { Sugested } \\
\text { species/Espécie } \\
\text { sugerida }\end{array}$ & $\begin{array}{c}\text { Accession number } \\
\text { /Número de acesso } \\
\text { (NCBI) }\end{array}$ & $\begin{array}{c}\text { Identity/Identi } \\
\text { dade (\%) }\end{array}$ \\
\hline ID029 & Monascus purpureus & AF033394.1 & 100 \\
\hline
\end{tabular}




\section{Discussion}

In the present study, DNA identification of Monascus purpureus NART001 strain was performed using DNA sequencing analysis for molecular species identification. The combination of the highest identity, total score and query cover values were used to attribute the suggested species. The obtained result showed similarity with a recent study (11) on identification using a Monascus purpureus isolate.

Monascus purpureus is a commercially important organism owing to its production of the beneficial compound monacolin $\mathrm{k}$ (12), which has been shown to have a favorable impact on lipid profiles of hypercholesterolemic patients.

Before initiating Monascus-fermented product production, is important to identify the species in order to obtain the complete metabolic profile, including pigments and monacolins.

Further studies on genes related to monacolin k biosynthesis from the Monascus purpureus NART001 strain could provide useful information on secondary metabolic processes and optimize it as a better source of monacolin $\mathrm{k}$.

\section{Conclusion}

In this study the identification of the strain, using a DNA sequencing analysis for molecular species identification were performed and results of investigation revealed that the Monascus purpureus NART001 strain showed 100\% identity with Monascus purpureus.

\section{Conflict of Interest}

The authors declare that there are no financial or personal relationships that could be understood as representing a potential conflict of interest.

\section{Discussão}

No presente estudo foi possível realizar a identificação do DNA da estirpe NART001 de Monascus purpureus, utilizando uma análise de sequenciamento de DNA para identificação de espécies moleculares. A combinação dos valores mais altos de identidade, pontuação total e consulta foi utilizada para atribuição das espécies sugeridas. O resultado obtido mostrou similaridade com um estudo recente (11) sobre identificação utilizando um isolado de Monascus purpureus.

Monascus purpureus é um organismo comercialmente importante devido à sua produção de monacolina $\mathrm{k}$ (12) responsável pelo impacto favorável no perfil lipidico de indivíduos dislipidémicos.

Antes de dar início à produção de produtos fermentados com Monascus é importante identificar as espécies para se obter o perfil de metabólitos adequado, como pigmentos e monacolinas.

Estudos a realizar num futuro próximo sobre genes relacionados com a biossíntese de monacolina $\mathrm{k}$ da estirpe NART001 de Monascus purpureus podem fornecer informações úteis sobre processos metabólicos secundários e optimizá-lo como um melhor recurso de monacolina $\mathrm{k}$.

\section{Conclusão}

Neste estudo, foi realizada a identificação da estirpe utilizando uma análise de sequenciação de DNA para identificação de espécies moleculares, e os resultados da investigação revelaram que a estirpe Monascus purpureus NART001 apresentou 100\% de identidade com Monascus purpureus.

\section{Conflito de interesses}

Os autores declaram não existir qualquer relação de natureza financeira ou pessoal que possa ser entendida ou representar um potencial conflito de interesses. 


\section{References/Referências}

[1] Lange, L., Bech, L., Busk, et al. (2012). The importance of fungi and of mycology for a global development of the bioeconomy. IMA Fungus : The Global Mycological Journal, 3(1), 87-92. http://doi.org/10.5598/imafungus.2012.03.01.09

[2] Dikshit R, Tallapragada P. Comparative study of Monascus sanguineus and Monascus purpureus as potential sources for red pigment production. International Journal of Pharma and Bio Sciences, 2012; 3: $885-895$.

[3] Marley E., Brown P., Leeman D. and Donnelly C., Analysis of citrinin in cereals, red yeast rice dietary supplement, and animal feed by immunoaffinity column cleanup and LC with fluorescence detection, Journal of AOAC Int., 2016; 99:1025-31.

[4] Li Y.G., Liu H., and Wang Z.T., A validated stability-indicating HPLC with photodiode array detector (PDA) method for the stress tests of Monascus purpureus fermented rice, red yeast rice, J. Pharm Biomed Anal., 2005; 39: 82-90.

[5] Nguyen T., Karl M., Santini A. Red Yeast Rice. Foods, 2017;(6)3.pii: E19. doi: 10.3390/foods6030019.

[6] Silva A., Moreira J., Andrade P., Nicolai M., Rijo P., Tavares N. Production of fermented Thai red glutinous rice using an isolated Monascus purpureus NART001 from commercially available Chinese red fermented rice. Biomed Biopharm Res, 2016;(13)2:201-208 DOI: 10.19277/bbr.13.2.138

[7] Harrington TC, Steimel J, Workneh F, Yang XB. Molecular identification of fungi associated with vascular discoloration of soybean in the North Central United States. Plant Diseases, 2000; 84: 83-89.

[8] Saad DS, Kinsey GC, Kim S, Gaylarde CC. Extraction of genomic DNA from filamentous fungi in biofilms on water-based paint coatings. International Journal of Biodeterioration and Biodegradation, 2004; 54: 99-103.

[9] Anderson IC, Campbell CD, Prosser JI. Potential bias of fungal 18S rDNA and internal transcribed spacer polymerase chain reaction primers for estimating fungal biodiversity in soil. Environmental Microbiology, 2003; 5: 36-47.

[10] Altschul, S.F., Gish, W., Miller, W., Myers, E.W., and Lipman, D.J. Basic local alignment search tool. J. Mol. Biol. 1990;215: 403-410.

[11] Srianta, I., Harijono. Monascus-fermented sorghum: pigments and monacolin K produced by Monascus purpureus on whole grain, dehulled grain and bran substrates. International Food Research Journal 2015; 22: 377-382.

[12] Zhang, C., Liang, J, Yang, Le. Novo RNA Sequencing and Transcriptome Analysis of Monascus purpureus and Analysis of Key Genes Involved in Monacolin K Biosynthesis. PLOS ONE 2017. DOI: https://doi.org/10.1371/journal.pone.0170149 